\title{
Expression of cluster of differentiation-95 and relevant signaling molecules in liver cancer
}

\author{
XUMING WANG $^{1,2,3^{*}}$, KANGLAI WEI $^{4^{*}}$, QIONGGUANG ZHANG $^{5}$, SIEN ZENG $^{1}$, \\ JING LIN $^{1}$, LI QIAO ${ }^{6}$ and LIJIANG LIU ${ }^{2,3}$ \\ ${ }^{1}$ Department of Pathology, Guilin Medical University, Guilin 541001; Departments of ${ }^{2}$ Pathology and Pathophysiology, \\ and ${ }^{3}$ Pathology, School of Medicine, Jianghan University, Wuhan Economy and Technology Development Zone, \\ Wuhan, Hubei 430056; ${ }^{4}$ Department of Pathology, The First Affiliated Hospital of Guangxi Medical University, \\ Nanning, Guangxi 530021; ${ }^{5}$ State Key Laboratory of Virology, National Laboratory of Antiviral and Tumor of \\ Traditional Chinese Medicine, Institute of Medical Virology, Research Center of Food and \\ Drug Evaluation, School of Medicine, Wuhan University, Wuhan, Hubei 430056; ${ }^{6}$ Office of \\ Graduate Student Affairs, Guilin Medical University, Guilin 541001, P.R. China
}

Received April 12, 2014; Accepted November 25, 2014

DOI: $10.3892 / \mathrm{mmr} .2014 .3129$

\begin{abstract}
The present study investigated the protein expression levels of cluster of differentiation (CD)95, caspase-8, caspase-3 and poly(ADP-ribose) polymerase 1 (PARP1) in liver cancer and its association with clinical pathological parameters. The results demonstrated that the expression of CD95 correlated with histological differentiation, liver cirrhosis, lymph node metastasis and distant metastasis $(\mathrm{P}<0.05)$, however, no correlations with gender, age, quantity of tumor nodules or $\mathrm{T}$ stage were observed ( $\mathrm{P}>0.05)$. The expression of CD95 was upregulated using a plasmid, which led to an increase in the expression levels of caspase- 8 and caspase- 3 and a decrease in the expression of PARP1. Upregulation of CD95 also promoted the apoptosis of the liver cancer cells. These results indicated that CD95 was associated with liver cancer and promoted the apoptosis of liver cancer cells by caspase-8, caspase-3 and PARP1.
\end{abstract}

\section{Introduction}

The cluster of differentiation (CD)95 antigen, also termed apoptosis antigen (APO)-1 or Fas, is a cell surface protein with a molecular weight of 200,000 Da, which differs to the molec-

Correspondence to: Dr Lijiang Liu, Department of Pathology and Pathophysiology, School of Medicine, Jianghan University, Building J12, 8 Sanjiaohu Road, Wuhan Economy and Technology Development Zone, Wuhan, Hubei 430056, P.R. China

E-mail: liulijiang@163.com

${ }^{*}$ Contributed equally

Key words: liver cancer, cluster of differentiation 95, caspase-8, caspase-3, poly(ADP-ribose) polymerase 1 ular weight of the tumor necrosis factor (TNF) receptor (1). Anti-CD95 induces cell-death and its activity is indistinguishable from the cytolytic activity of TNF (1). A previous study observed that anti-CD95 induces apoptosis in vivo. Nanogram quantities of anti-CD95 completely inhibited the proliferation of cells containing APO-1 in vitro, characteristic of the process of programmed cell death or apoptosis (2). Complementary DNA (cDNA) encoding the Fas cell surface antigen were isolated from a cDNA library of human T-cell lymphoma KT-3 cells by Itoh et al (3) The nucleotide sequence of the cDNA revealed that the molecule coding for the Fas antigen determinant is a 319 amino acid polypeptide with a single transmembrane domain. The extracellular domain is rich in cysteine residues and is similar to that of human TNF receptors, the human nerve growth factor receptor and the CD40 human B cell antigen $(3,4)$.

Apoptosis is the predominant form of eukaryotic cell death and occurs during tissue replacement, organ development, metamorphosis, tissue atrophy and tumor regression (4-6). Apoptosis is induced by a diverse range of agents, including glucocorticoids, cytostatic drugs, cytolytic cytokines, including TNF and lymph toxin, and in target cells of various killer cells, including cytotoxic T lymphocytes (4-6). The most prominent morphological features of apoptosis are chromatin condensation and membrane blebbing (4-6). In cells undergoing apoptosis, an endonuclease is induced, which cleaves the genomic DNA into polynucleosomal fragments and is observed on agarose gels as a 'DNA ladder' (4). Apoptosis and the Fas system are important in the process of converting liver cirrhosis into hepatocellular carcinoma. Downregulation in the expression of Fas and upregulation in the expression of Fas ligand in hepatocytes, and elevation of serum levels of Fas are important in tumor evasion from immune surveillance and in hepatic carcinogenesis $(5,6)$. The present study investigated the relative expression of CD95 in liver cancer cells to determine whether there is a link between CD95 and liver cancer. 


\section{Materials and methods}

Reagents. The rabbit polyclonal immunoglobulin $\mathrm{G}(\mathrm{IgG})$ anti-CD95 antibody (N-18; cat. no. sc-714), mouse monoclonal $\mathrm{IgG}_{1}$ anti-caspase-8 antibody (1.1.40; cat. no. sc-81656), rabbit polyclonal $\operatorname{IgG}$ anti-caspase-3 antibody (H-277; cat. no. sc-7148), goat polyclonal IgG anti-poly(ADP-ribose) polymerase 1 (PARP1) antibody (A-20; cat. no. sc-1562) and mouse monoclonal $\mathrm{IgG}_{1}$ anti- $\beta$-actin antibody $(\mathrm{C} 4$; cat. no. sc-47778) were purchased from Santa Cruz Biotechnology, Inc. (Santa Cruz, CA, USA). The radioimmunoprecipitation buffer and enhanced bicinchoninic acid assay kit were purchased from Beyotime Institute of Biotechnology (Jiangsu,China). Polyvinylidene difluoride (PVDF) membranes were purchased from EMD Millipore (Billerica, MA, USA). The LipoFiter ${ }^{\mathrm{TM}}$ Liposomal Transfection reagent was purchased from HanBio (Shanghai, China). The SP test kit and diaminobenzidine (DAB) colorization test kit were purchased from Beijing Zhongshan Golden Bridge Biotechnology, Co., Ltd. (Beijing, China). The AnnexinV-fluorescein isothiocyanate (FITC)/propidium iodide (PI) apoptosis detection kit was purchased from KeyGen Biotech, Co., Ltd. (Nanjing, China).

Human liver cancer tissues. Frozen tumor samples and the corresponding normal liver tissues were obtained from 40 patients with gastric cancer, between 2006 and 2012 at Guangxi Medical University (Nanning, Guangxi, China) and paraffinembedded samples were obtained from 66 patients with gastric cancer, between 2006 and 2012 at Guangxi Medical University, Guilin Medical University, Guilin, Guangxi, China and Jianghan University, Wuhan, Hubei, China. Written informed consent was obtained from all patients. These samples were used following approval of the Institutional Review Board. The study was approved by the ethics committee of the School of Medicine, Jianghan University (Wuhan, China).

Cell lines. The human HepG2 liver cancer cell line and the SGC7901 cell line were obtained from the Cell Center of Basic Medicine, Chinese Academy of Medical Sciences (Beijing, China).

CD95 expression plasmid construction. The CD95 expression plasmid was donated by Dr JunFeng Zhang (Institute of Genetics and Developmental Biology, Chinese Academy of Sciences, Beijing, China). The total RNA was extracted from the SGC7901 cell line using TRIzol reagent (Invitrogen Life Technologies, Carlsbad, CA, USA) and the first strand cDNA was synthesized by reverse transcription using a ReverAid First Strand cDNA Synthesis kit (Thermo Fisher Scientific, Waltham, MA, USA) at $42^{\circ} \mathrm{C}$, according to the manufacturer's instructions. The entire CD95 coding region was amplified using the following primers: CD95, forward 5'-cactcgagCTTTCACTTCGGAGGATTGC-3' (the added XhoI site is lowercase) and reverse 5'-gtgaattctGACCAAGCTTTGGATTTCATTTC-3' (the added EcoRI site is underlined). The primers were designed using Primer 5.0 (Primer Biosoft International, Palo Alto, CA, USA) and were synthesized by the Sangon Biotech Co., Ltd. (Shanghai, China). Polymerase chain reactions were performed using a Ready-To-Use PCR kit (Tag DNA polymerase; no. SK2082, Sangon Biotech Co., Ltd) as follows: $94^{\circ} \mathrm{C}$ for $4 \mathrm{~min}, 30$ cycles of $94^{\circ} \mathrm{C}$ for $30 \mathrm{sec}$, $60^{\circ} \mathrm{C}$ for $30 \mathrm{sec}$ and $72^{\circ} \mathrm{C}$ for $70 \mathrm{sec}$ with a final extension at $72^{\circ} \mathrm{C}$ for $10 \mathrm{~min}$. The resulting fragment was digested using $\mathrm{XhoI}$ and EcoRI (Sangon Biotech Co., Ltd) and then subcloned into the pEgreen fluorescent protein (GFP)-N1, resulting in the N-terminal fusion to GFP. The resulting construct, pCMV IE: CD95-enhanced GFP (CD95-vector), was sequenced to confirm the in-frame fusion of CD95 and GFP and was used for transient expression in the HepG2 cells.

Cell culture. All the cells were maintained in RPMI-1640 medium containing $10 \%$ fetal bovine serum (FBS; Invitrogen Life Technologies) at $37^{\circ} \mathrm{C}$ in a $5 \% \mathrm{CO}_{2}$ atmosphere.

Cell transfection. All the transfections were performed using LipoFiter ${ }^{\mathrm{TM}}$ Liposomal Transfection reagent, according to the manufacturer's instructions. The HepG2 cells were plated (6x $10^{6}$ cells) in $100 \mathrm{~mm}$ dishes and incubated overnight prior to replacing with fresh RPMI-1640 medium supplemented with $10 \%$ FBS. The cells were transfected with $4.6 \mu \mathrm{g}$ of either CD95-vector or vector-control using $4.8 \mu \mathrm{l}$ LipoFiter $^{\mathrm{TM}}$ Liposomal Transfection reagent. The media was replaced $6 \mathrm{~h}$ after transfection and the cells were incubated at $37^{\circ} \mathrm{C}$ for $48 \mathrm{~h}$. pEGFP-N1 was used as the vector-control. These transfected cells were used for subsequent experiments.

Immunohistochemistry. Immunohistochemical staining was performed on $5 \mu \mathrm{m}$-thick tumor sections using a 'two-step' method. The tissue slides were de-paraffinized with $100 \%$ xylene for $10 \mathrm{~min}$ and rehydrated gradually in an alcohol series. The endogenous peroxidase activity was inhibited by incubation in a $3 \%$ hydrogen peroxide/methanol buffer for $10 \mathrm{~min}$. Antigen retrieval was performed by immersing the slides in $0.5 \mathrm{~mol} / \mathrm{l}$ ethylenediamine tetraacetic acid buffer (pH 8.0) for $10 \mathrm{~min}$, followed by boiling in a waterbath for $25 \mathrm{~min}$. The slides were rinsed in phosphate-buffered saline (PBS) and subsequently incubated with polyclonal anti-CD95 antibody (1:200) overnight at $4^{\circ} \mathrm{C}$ in a humidified chamber. Following incubation, the slides were washed three times with PBS containing $0.05 \%$ Tween- 20 for 2 min each time. The slides were then incubated with $100 \mu 1$ horseradish peroxidase polymer-anti-Mouse/Rabbit immunoglobulin G covering the tissue section in a moist chamber for $15 \mathrm{~min}$. The slides were then washed three times, as previously, and the tissue was incubated for 5 min with $100 \mu \mathrm{l}$ DAB chromogen. Following development of the appropriate color, the slides were washed gently under tap water for $\sim 1-2$ min, prior to counterstaining with $100 \mu 1$ Mayer's hematoxylin (Sangon Biotech Co., Ltd) covering the tissue completely for $\sim 20 \mathrm{sec}$. The slides were rinsed thoroughly with tap water for $\sim 1-2$ min and subsequently incubated in PBS for 20 sec until the color turned blue. The slides were then rinsed with distilled water, followed by tap water.

The levels of CD95 staining were scored as follows: 0 , no staining or staining observed in $<10 \%$ tumor cells; $1+$, faint/barely perceptible staining detected in $\geq 10 \%$ tumor cells; $2+/ 3+$, moderate/strong staining, respectively, observed in $\geq 10 \%$ tumor cells. A score of $0 / 1+$ was considered negative and a score of $2+/ 3+$ was considered positive. The immunos- 
Table I. Association between the expression of CD95 and the clinicopathological features of liver cancer.

\begin{tabular}{|c|c|c|c|}
\hline \multirow[b]{2}{*}{ Factor } & \multicolumn{2}{|c|}{ Expression of CD95 } & \multirow[b]{2}{*}{ P-value } \\
\hline & Positive (n) & Negative (n) & \\
\hline \multicolumn{4}{|l|}{ Gender } \\
\hline Male & 10 & 31 & \multirow{2}{*}{0.74} \\
\hline Female & 7 & 18 & \\
\hline \multicolumn{4}{|l|}{ Age (years) } \\
\hline$\leq 50$ & 5 & 16 & \multirow{2}{*}{0.80} \\
\hline$>50$ & 12 & 33 & \\
\hline \multicolumn{4}{|c|}{ Histological differentiation } \\
\hline High & 12 & 13 & \multirow{3}{*}{0.002} \\
\hline Medial & 2 & 8 & \\
\hline Low & 3 & 28 & \\
\hline \multicolumn{4}{|c|}{ Tumor nodules } \\
\hline Single & 6 & 20 & \multirow{2}{*}{0.68} \\
\hline Multiple & 11 & 29 & \\
\hline \multicolumn{4}{|c|}{ Liver cirrhosis } \\
\hline Positive & 8 & 37 & \multirow{2}{*}{0.029} \\
\hline Negative & 9 & 12 & \\
\hline \multicolumn{4}{|l|}{ T stage } \\
\hline $\mathrm{T} 1$ & 5 & 7 & \multirow{4}{*}{0.064} \\
\hline $\mathrm{T} 2$ & 6 & 11 & \\
\hline $\mathrm{T} 3$ & 4 & 21 & \\
\hline $\mathrm{T} 4$ & 2 & 10 & \\
\hline \multicolumn{4}{|l|}{$\mathrm{N}$ stage } \\
\hline No & 13 & 19 & \multirow{2}{*}{0.016} \\
\hline N1-3 & 4 & 30 & \\
\hline \multicolumn{4}{|l|}{ M stage } \\
\hline M0 & 16 & 32 & \multirow{2}{*}{0.047} \\
\hline M1 & 1 & 17 & \\
\hline
\end{tabular}

$\mathrm{CD}$, cluster of differentiation; $\mathrm{n}$, number of cells.

tained slides were evaluated independently by two pathologists in a blinded-manner. In the majority of cases, the results of the evaluation the two pathologists were identical; discrepancies were resolved by re-examination and consensus.

Western blot analysis. For western blot analysis, the cells were washed with cold PBS and lysed in a lysis buffer containing $50 \mathrm{mM}$ Tris- $\mathrm{HCl}$ (pH 8.0), $150 \mathrm{mM} \mathrm{NaCl}, 0.25 \mathrm{mM}$ EDTA (pH 8.0), 0.1\% SDS, $1 \%$ Triton $\mathrm{X}-100$ and $50 \mathrm{mM} \mathrm{NaF}$, supplemented with MS-SAFE ${ }^{\mathrm{TM}}$ Protease and Phosphatase Inhibitor Cocktail (1:100; Sigma-Aldrich, St. Louis, MO, USA) and phosphatase inhibitors (Sigma-Aldrich). The protein concentrations were determined using an Enhanced Bicinchoninic Acid Protein Assay kit (Beyotime Institute of Biotechnology). The cell lysates were mixed with loading buffer (Beyotime Institute of Biotechnology), separated using 12\% SDS-PAGE gels and transferred onto a PVDF membrane (EMD Millipore, Billerica, MA, USA). The membranes were subsequently probed with various primary antibodies, appropriate secondary antibodies [goat anti-mouse IgG-horseradish peroxidase (HRP; sc-2005), goat anti-rabbit IgG-HRP (sc-2004) and donkey anti-goat IgG-HRP (sc-2020)] and visualized using enhanced chemiluminescence detection reagents (DNR Bio-Imaging Systems, Ltd., Jerusalem, Israel). The density of the protein bands were assessed using Totallab analysis software, version 2.01 (Nonlinear USA, Inc., Durham, NC, USA).

Apoptotic assay. The cells were stained using an Annexin V-FITC apoptosis detection kit (Nanjing KeyGen Biotech, Co., Ltd., Nanjing, China) according to the manufacturer's instructions, to detect early apoptotic cells (Annexin V+PIevents) and necrotic or late apoptotic cells (Annexin V+PI+ events) by flow cytometry. Briefly, the HepG2 cells were transfected with either the vector-CD95 or vector-control for $48 \mathrm{~h}$. The cells were then collected and resuspended in the culture medium at a density of $1 \times 10^{6}$ cells $/ \mathrm{ml}$, stained with $5 \mu \mathrm{L}$ Annexin V-FITC and $5 \mu \mathrm{L}$ PI in $300 \mu \mathrm{L}$ binding 
Table II. Expression of CD95 is associated with the expression of caspase-8, caspase-3 and PARP1.

\begin{tabular}{lccr}
\hline & \multicolumn{2}{c}{ Expression of CD95 } & K-value/P-value \\
\cline { 2 - 3 } Factor & Positive (n) & Negative (n) & $\kappa=0.78$ \\
\hline Caspase-8 & 12 & 2 & $\mathrm{P}<0.01$ \\
Positive & 2 & 24 & $\kappa=0.609$ \\
Negative & 10 & 3 & $\mathrm{P}<0.01$ \\
Caspase-3 & 4 & 23 & $\mathrm{P}<0.01$ \\
Positive & & & \\
Negative & 3 & 25 & \\
PARP1 & 11 & 1 & \\
Positive & & & \\
Negative & & & \\
\hline
\end{tabular}

$\mathrm{CD}$, cluster of differentiation; PARP1, poly(ADP-ribose) polymerase 1; n, number of cells.

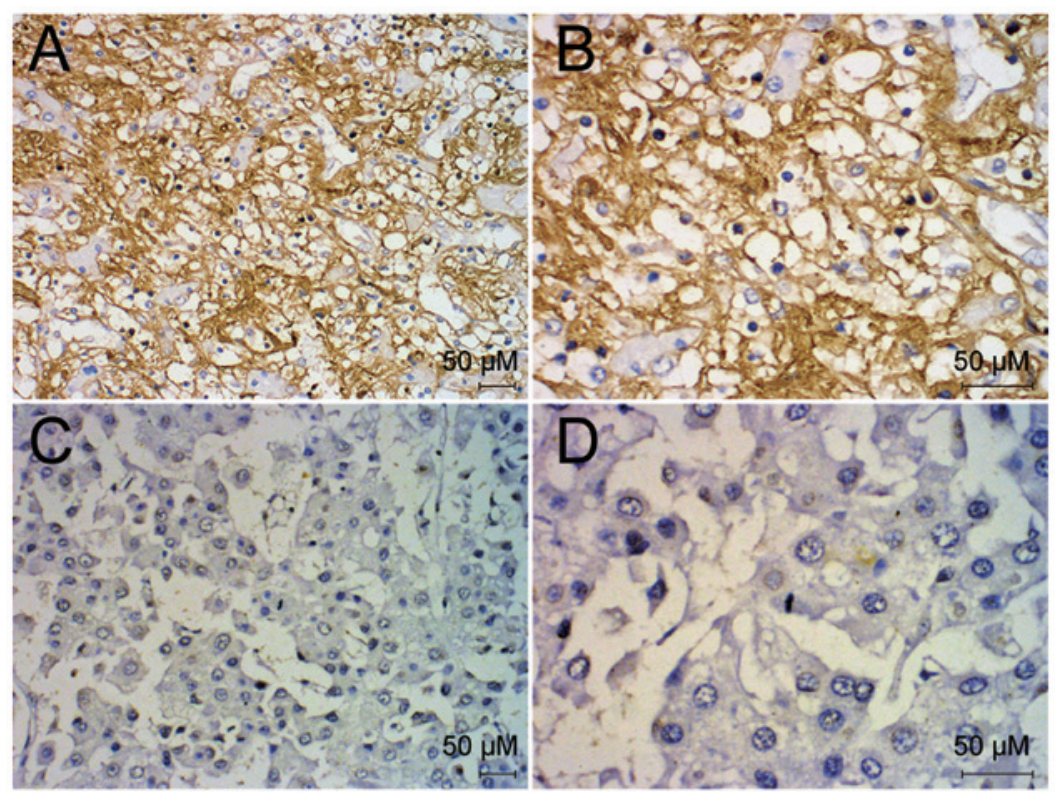

Figure 1. Immunohistochemical staining of liver cancer tissues. Representative images of the positive and negative CD95 staining. (A) Positive CD95 staining in the liver cancer cell membrane (magnification, x100). (B) Positive CD95 staining in the liver cancer cell membrane (magnification, x400). (C) Negative CD95 staining in the liver cancer cell membrane (magnification, x100). (D) Negative CD95 staining in the liver cancer cell membrane (magnification, x400).

buffer containing $10 \mathrm{mM}$ HEPES, (pH 7.4), $140 \mathrm{mM} \mathrm{NaOH}$ and $2.5 \mathrm{mM} \mathrm{CaCl}{ }_{2}$ according to the manufacturer's instructions for $15 \mathrm{~min}$ at room temperature in the dark. Quantification of the apoptotic cells was assessed using a FACScan flow cytometer (Beckman Coulter, Brea, CA, USA).

Statistical analysis. Statistical analysis was performed using SPSS 12.0 software (SPSS, Inc., Chicago, IL, USA). The data are expressed as the mean \pm standard deviation of three replicates and were compared using Student's t-test and analysis of variance. $\mathrm{P}<0.05$ was considered to indicate a statistically significant difference. All experiments were performed at least three times to ensure reproducibility of the results.

\section{Results}

Association between the expression of CD95 and clinicopathological features in liver cancer. The expression of CD95 was examined in the 66 liver cancer samples using immunohistochemistry. The expression of CD95 was detected predominantly in the cytoplasm of the liver cancer cells and at the plasma membrane, however, no expression was detected in the nuclei (Fig. 1). Positive expression of CD95 was detected in 17 of the liver cancer samples. The expression of CD95 correlated with histological differentiation, liver cirrhosis, lymph node metastasis and distant metastasis $(\mathrm{P}<0.05)$, however, no correlations with gender, age, quantity of tumor nodules or T stage were observed ( $\mathrm{P}>0.05$; Table I). 


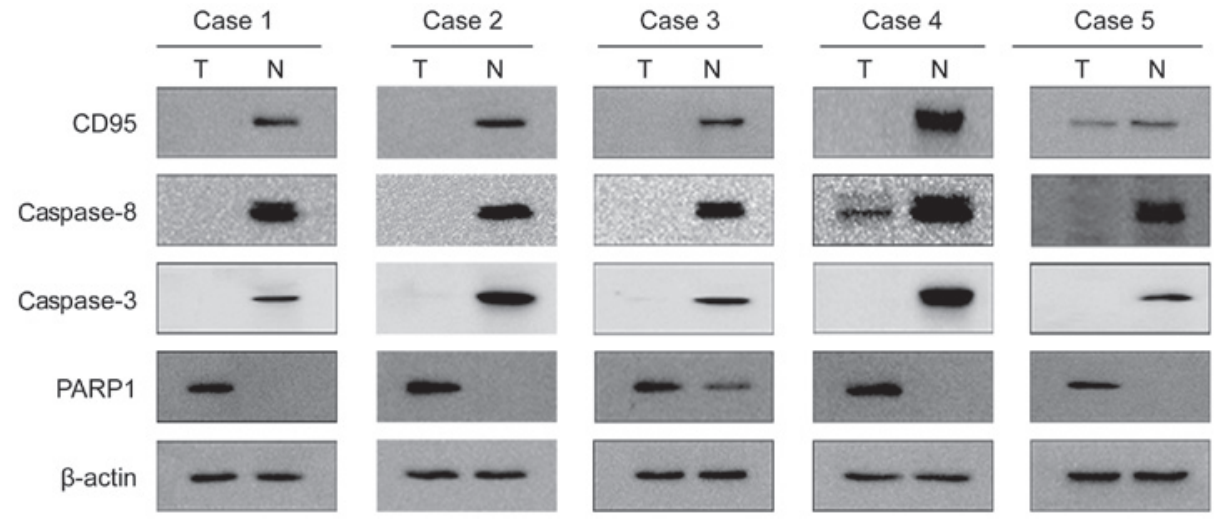

Figure 2. Western blot analysis of the expression levels of CD95, caspase-8, caspase-3 and PARP1 in specimens from liver cancer tissues and corresponding normal tissues. A representative western blot analysis result of five samples is shown. $\mathrm{CD}$, cluster of differentiation; PARP, poly ADP ribose polymerase, $\mathrm{T}$, liver cancer tissues; $\mathrm{N}$, normal tissues.

\section{A}
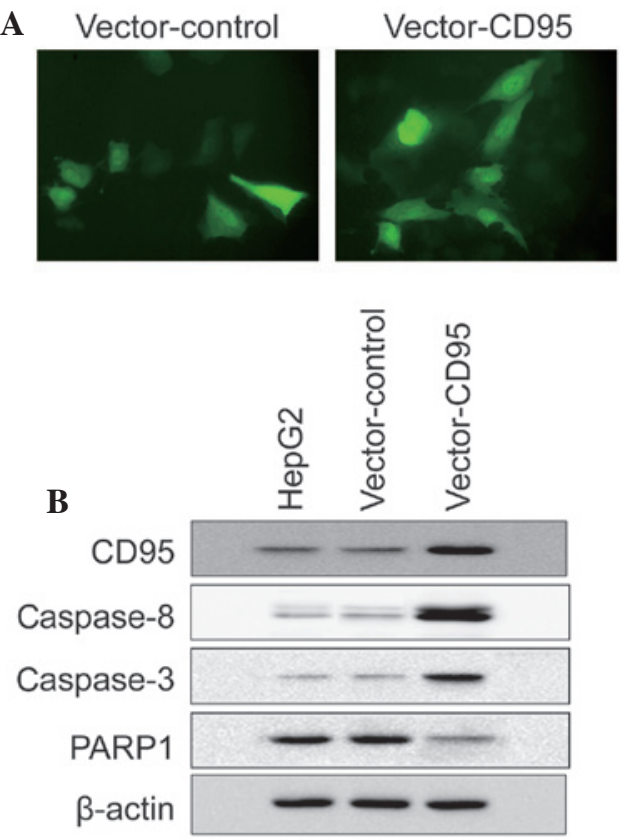

\section{C}

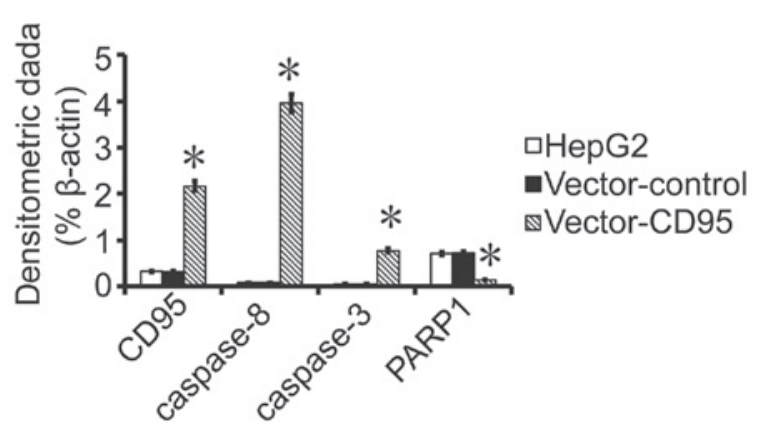

Figure 3. (A) Green fluorescence was observed at $24 \mathrm{~h}$ in the vector-control cells and the vector-CD95 cells (magnification, $\mathrm{x} 400$ ). (B and C) Western blot analysis of the expression levels of CD95, caspase-8, caspase-3 and PARP1 in the recombinant liver cancer cell line. The data are expressed as the mean \pm standard deviation from three independent experiments $\left({ }^{*} \mathrm{P}<0.05\right.$, compared with HepG2). CD, cluster of differentiation; PARP, poly ADP ribose polymerase.

Expression of CD95 is associated with the expression levels of caspase- 8 , caspase- 3 and PARPI. The protein expression levels of CD95, caspase-8, caspase-3 and PARP1 in the tumor
A

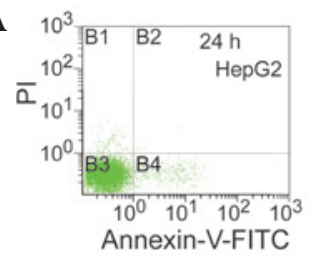

C

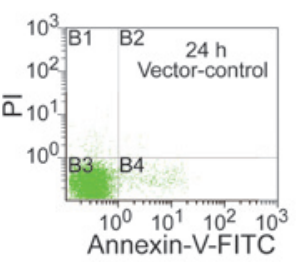

$\mathbf{E}$
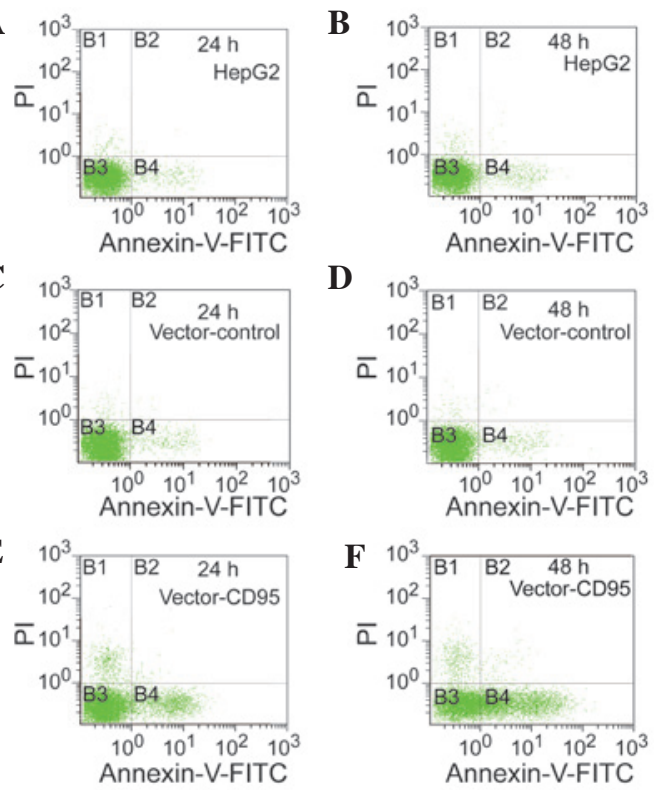

$\mathbf{D}$
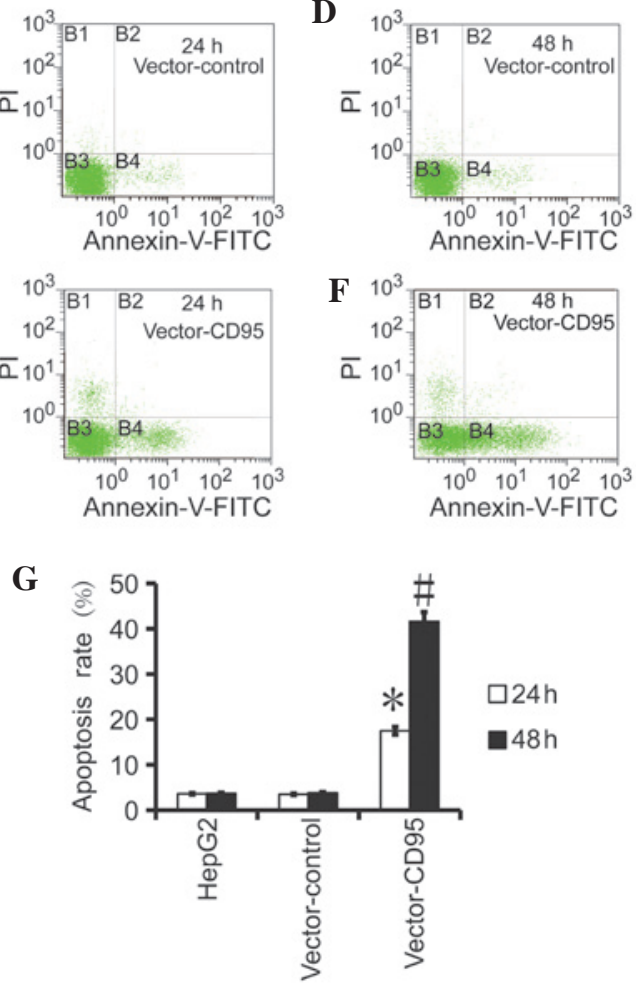

Figure 4. Apoptotic rate detected by flow cytometry. (A) Apoptotic rate at $24 \mathrm{~h}$ in the HepG2 cells. (B) Apoptotic rate of the HepG2 cells at 48 h. (C) Apoptotic rate of the thevector-control cells at $24 \mathrm{~h}$ and (D) $48 \mathrm{~h}$. (E) Apoptotic rate of the vector-CD95 cells at $24 \mathrm{~h}$ and (F) $48 \mathrm{~h}$. (G) Statistical analysis of the apoptotic rate in the HepG2 and recombinant liver cancer cell line. The data are expressed as the mean \pm standard deviation from three independent experiments ( $\mathrm{P}<0.05$ compared with the HepG2 cells). PI, propidum iodide; FITC, fluorescein isothiocyanate, $\mathrm{CD}$, cluster of differentiation.

tissues and the corresponding normal tissues of 40 liver cancer samples were assessed by western blot analysis. The positive expression of CD95, caspase- 8 and caspase-3 was detected in 
$14(35 \%), 14(35 \%)$ and $13(32.5 \%)$ of the 40 tumor specimens, respectively. These levels were lower compared with those in the normal tissues, in which the positive expression of CD95, caspase- 8 and caspase-3 was observed in 32 (80\%), 33 (75\%) and $36(90 \%)$ specimens, respectively (Fig. 2). The expression of PARP1 was detected in $28(70 \%)$ tumor specimens, compared with $16(40 \%)$ in the normal liver tissue specimens (Fig. 2). The expression of CD95 correlated with the expression of caspase-8, caspase-3 and PARP1 in the tumor specimens (Table II, $\mathrm{P}<0.01$ ).

Cell transfection. A positive GFP signal was detected in the High95 cells and in the control cells $24 \mathrm{~h}$ after transfection, which indicated that the transfection was successful (Fig. 3). Western blot analysis revealed that the expression of CD95 was higher in the High95 cells compared with the HepG2 cells and the control cells $(\mathrm{P}<0.05$; Fig. 3). No difference was observed between the HepG2 cells and the control cells.

Expression levels of CD95, caspase-8, caspase-3 and PARP1. Western blot analysis indicated that the expression levels of CD95, caspase- 8 and caspase- 3 were higher in the High95 cells compared with the HepG2 cells and control cells $(\mathrm{P}<0.05$; Fig. 4). The western blot analysis results also revealed that the expression of PARP1 was lower in the High95 cells compared with the HepG2 cells and control cells( $\mathrm{P}<0.05$; Fig. 4). No difference was observed in the expression levels of CD95, caspase- 8 , caspase-3 and PARP between the HepG 2 cells and control cells.

CD95 promotes apoptosis in the HepG2 cells. The results of the flow cytometry revealed that the level of apoptosis in the High95 cells was higher compared with the HepG2 cells and the control cells (Fig. 5). Furthermore, the apoptotic rate was higher at $48 \mathrm{~h}$ compared with $24 \mathrm{~h}$, which indicated that CD95 mediated apoptosis in the HepG2 cells.

\section{Discussion}

CD95 is widely expressed in normal and diseased tissues and has been implicated in tumor progression in several types of cancer (5). Reduced expression levels of CD95 have been observed in a number of tumor types(7-12). The present study demonstrated that the expression of CD95 was lower in liver cancer tissues compared with normal liver tissues and correlated with histological differentiation, liver cirrhosis, lymph node metastasis and distant metastasis $(\mathrm{P}<0.05)$. Conversely, no correlations were observed with gender, age, quantity of tumor nodules or $\mathrm{T}$ stage, indicating that the expression of CD95 was associated with liver cancer.

In the present study, western blot analysis revealed that the expression of CD95 correlated with the expression levels of caspase-8, caspase-3 and PARP1. Caspase- 8 is important in the CD95-mediated activation of the mitogen-activated protein kinases (MAPKs). A mechanism has been proposed, in which the catalytic activity and substrate specificity of caspase- 8 are determined by the conformation and cleavage status of procaspase-8 (13). Procaspase- 8 processing is required for the CD95-induced activation of MAPKs and conditions impairing MAPK activation are accompanied by reduced procaspase- 8 processing (14). The activation of procaspase- 8 is hypothesized to occur through an 'induced proximity' mechanism, involving the dimerization of procaspase- 8 molecules, which facilitates activation through subsequent self-processing. This is in contrast to the executioner caspases, caspase-3 and caspase-7, which are constitutively dimeric and inactive due to the 'strain' caused by their short interdomain linker region on the active site and only become active on proteolytic cleavage (13). CD95 may rely exclusively on the activation of caspase- 8 and the mitochondrial activation of caspase-3, which can process more procaspase- 8 and, thus, propagate the amplification of the apoptotic signal (15). The present study revealed that the expression of caspase- 8 was lower in the liver cancer tissues compared with the normal liver tissues.

Normal cells contain only a small quantity of caspases, in the form of inactive zymogens, and activated caspases are transformed to proteases via the catalytic activity of enzymes, which are capable of cleaving a number of substrate proteins resulting in apoptosis $(16,17)$. Caspase- 3 is activated by a series of cascade reactions until DNase is activated, which belongs to the $\mathrm{Mg}^{2+}$-dependent endonucleases and acts as an apoptotic factor (16). As caspase-3 is an effector caspase in apoptotic pathways, previous studies have hypothesized that a loss in the expression of caspase-3 may be important in the carcinogenesis of hepatocellular carcinoma (17). The present study revealed that the expression of caspase- 3 was lower in the liver cancer tissues compared with the normal liver tissues.

PARP1 is a nuclear enzyme, which catalyzes PARP in target proteins in response to DNA damage and is considered to be important in DNA repair/recombination, cell death, cell proliferation and for stabilization of the genome (18). In several types of cancer, the expression of PARP1 is high and, therefore, inhibiting the expression of PARP1 may improve outcomes in patients with cancer (19-22). The present study demonstrated that the expression of PARP1 was higher in the liver cancer tissues compared with the normal liver tissues. In addition, the expression of PARP1 decreased as the expression of CD95 increased. The results demonstrated that inhibition of the expression of PARP1 may improve outcomes in patients with liver cancer.

\section{Acknowledgements}

This study was supported by the National Natural Science foundation of Guangxi (no. 0848014), the National Natural Science Foundation of China (no. 30870981), the Jianghan University Doctor Foundation (no. 1010-08110001) and the Science Foundation of Health Office of Hubei Province (no. NX200727).

\section{References}

1. Yonehara S, Ishii A and Yonehara M: A cell-killing monoclonal antibody (anti-Fas) to a cell surface antigen co-downregulated with the receptor of tumor necrosis factor. J Exp Med 169: 1747-1756, 1989.

2. Trauth BC, Klas C, Peters AM, et al: Monoclonal antibody-mediated tumor regression by induction of apoptosis. Science 245: 301-305, 1989.

3. Itoh N, Yonehara S, Ishii A, et al: The polypeptide encoded by the cDNA for human cell surface antigen Fas can mediate apoptosis. Cell 66: 233-243, 1991. 
4. Oehm A, Behrmann I, Falk W, et al: Purification and molecular cloning of the APO- 1 cell surface antigen, a member of the tumor necrosis factor/nerve growth factor receptor superfamily. Sequence identity with the Fas antigen. J Biol Chem 267: 10709-10715, 1992.

5. Hammam O, Mahmoud O, Zahran M, et al: The role of fas/fas ligand system in the pathogenesis of liver cirrhosis and hepatocellular carcinoma. Hepat Mon 12: e6132, 2012.

6. El Bassiouny AE, El-Bassiouni NE, Nosseir MM, et al: Circulating and hepatic Fas expression in $\mathrm{HCV}$-induced chronic liver disease and hepatocellular carcinoma. Medscape J Med 10: 130, 2008.

7. Lee YB, Kyung Kim E, Park HJ, et al: Expression of Fas and Fas ligand in primary cutaneous squamous cell carcinoma in association with grade of tumor differentiation. Int J Dermatol 52 1092-1097, 2013.

8. Sjostrom-Mattson J, Von Boguslawski K, Bengtsson NO Mjaaland I, Salmenkivi K and Blomqvist C: The expression of p53, bcl-2, bax, fas and fasL in the primary tumor and lymph node metastases of breast cancer. Acta Oncol 48: 1137-1143, 2009.

9. Bebenek M, Dus D and Kozlak J: Fas expression in primary breast cancer is related to neoplastic infiltration of perilymphatic fat. Adv Med Sci 53: 49-53, 2008.

10. Yang D, Torres CM, Bardhan K, Zimmerman M, McGaha TL, and Liu K: Decitabine and vorinostat cooperate to sensitize colon carcinoma cells to Fas ligand-induced apoptosis in vitro and tumor suppression in vivo. J Immunol 188: 4441-4449, 2012.

11. Yu Y, Bae S, Kim H, et al: The anti-tumor activity of vitamin C via the increase of Fas (CD95) and MHC I expression on human stomach cancer cell line, SNU1. Immune Netw 11: 210-215, 2011.

12. Volkmann M, Schiff JH, Hajjar Y, et al: Loss of CD95 expression is linked to most but not all p53 mutants in European hepatocellular carcinoma. J Mol Med (Berl) 79: 594-600, 2001.
13. Hughes MA, Harper N, Butterworth M, Cain K, Cohen GM and MacFarlane M: Reconstitution of the death-inducing signaling complex reveals a substrate switch that determines CD95-mediated death or survival. Mol Cell 35: 265-279, 2009.

14. Kober AM, Legewie S, Pforr C, et al: Caspase- 8 activity has an essential role in CD95/Fas-mediated MAPK activation. Cell Death Dis 2: e212, 2011.

15. Bajt ML, Vonderfecht SL and Jaeschke H: Differential protection with inhibitors of caspase- 8 and caspase- 3 in murine models of tumor necrosis factor and Fas receptor-mediated hepatocellular apoptosis. Toxicol Appl Pharmacol 175: 243-252, 2001.

16. Xiao LJ, Zhao S, Zhao EH, et al: Clinicopathological and prognostic significance of $\mathrm{Ki}-67$, caspase-3 and p53 expression in gastric carcinomas. Oncol Lett 6: 1277-1284, 2013.

17. Sun BH, Zhang J, Wang BJ, et al: Analysis of in vivo patterns of caspase 3 gene expression in primary hepatocellular carcinoma and its relationship to p21(WAF1) expression and hepatic apoptosis. World J Gastroenterol 6: 356-360, 2000

18. Lindahl T, Satoh MS, Poirier GG and Klungland A: Post-translational modification of poly(ADP-ribose) polymerase induced by DNA strand breaks. Trends Biochem Sci 20: 405-411, 1995.

19. Bi FF, Li D and Yang Q: Hypomethylation of ETS transcription factor binding sites and upregulation of PARP1 expression in endometrial cancer. Biomed Res Int 2013: 946268, 2013.

20. Wu W, Kong Z, Duan X, et al: Inhibition of PARP1 by small interfering RNA enhances docetaxel activity against human prostate cancer PC3 cells. Biochem Biophys Res Commun 442: 127-132, 2013

21. He W, Liu T, Shan Y, Zhu K and Li Y: PARP1 polymorphisms increase the risk of gastric cancer in a Chinese population. Mol Diagn Ther 16: 35-42, 2012.

22. Kim J, Pyun JA, Cho SW, Lee K and Kwack K: Lymph node metastasis of gastric cancer is associated with the interaction between poly (ADP-ribose) polymerase 1 and matrix metallopeptidase 2. DNA Cell Biol 30: 1011-1017, 2011. 